\title{
A Review on Contextualizing Health Care for Adolescents living with HIV
}

\author{
Ramatu Agambire \\ Department of Nursing, Faculty of Health Science, \\ Garden City University College, Kumasi Ghana \\ Cecilia Ackon Ansong \\ Department of Nursing, Faculty of Health Science, \\ Garden City University College, Kumasi Ghana
}

\begin{abstract}
Adolescents Living with HIV (ALHIV) grow up in circumstances quite different from those of their other no-infected counterparts with both psychological, economic, social and sexuality challenges and vulnerabilities. They have an urgent need for improved approaches to address their specific health care needs. Improving outcomes for adolescents and reaching global targets for an AIDS FREE generation by 2030 will require evidence-based interventions and policies. Howbeit, there is a growing area of research in HIV where a considerable amount of efforts is still needed to inform the understanding of what works for this population. Evidence indicates higher rates of loss to follow up, and poor adherence, as well as increased needs for psychosocial support among ALHIV. This population group continues to be underserved by current HIV services and have significantly worse access to and coverage of ART. A number of care models have been designed over the years to improve health outcomes among this group of population. Effective establishment of HIV treatment demands that HIV diagnosis is followed by timely linkage to outpatient care, prompt initiation of antiretroviral therapy and prophylactic medications when indicated, and subsequent adherence to prescribed medications as revealed in individual studies. Certainty of uptake and successful outcomes demands that, patients must progress through a sequence of steps taking into account environmental, patients predisposition, perceived and enabling factors as well as health behaviour models
\end{abstract}

Keyword: Adolescents, models, context, poor adherence, HIV services, anti-retroviral therapy.

\section{INTRODUCTION}

This review focuses on Adolescent Living with HIV (ALHIV). Adolescent has been defined as a translational stage that bridges the gap between childhood and adulthood [1]. According to literature, adolescent has been described as the period where most people develop biological, psychological, sexuality and psycho-social sensitivity [2]. Adolescence is typically a period of experimentation, new experiences, and vulnerability. As a result of sexuality exploitation associated with adolescents, access to sexual and reproductive health information and services has been prioritized and has become increasingly important [3]. Despites the recognized need for protection from HIV infections and other reproductive health risks, their age, social and economic status limit adolescent access to information and services in that context [4]. Sexually active life as part of adolescence continues with a high burden of HIV, therefore adolescence offers a window of opportunity to intervene early. Comprehensive data are vital to modelling accurate HIV-related messages and services before risky behaviours are formed and become entrenched [4]. 
Numerous global declarations and commitments, with specific goals and targets, have been made and set by world leaders and governments to respond to the HIV and AIDS epidemic since 2000 [5]. Most of the management policies and goals regarding adolescents are general in content, however; the United Nations General Assembly Special Session on HIV and AIDS (UNGASS) reports in 2001 explicitly included a target to reduce the prevalence of HIV in young people aged 15-24 years globally by $25 \%$ targeted in 2010 and to increase young people's access to crucial HIV prevention information, skills, and services in other to reach 95\% of those in need [5]. In 2015, a report by the UN Secretary General's High-Level Advisory Panel on the Post Millennium Development Goals Agenda emphasized equity, empowerment, and engagement of adolescents and youth in strengthening of data as central drivers of revolution in their next development agenda [6].

Despite the goals, targets and global commitments for adolescents, the implications and accountabilities are rarely adolescents-targeted. Also, adolescent-specific data are limited which incurs a serious barrier to measuring and monitoring progress. Although the international reporting process recommends reporting on disaggregated data on adolescents and youth, little of these data are published in global or national progress reports [7a]. Consequently, compared with infants and adults, less is known about the burden of HIV and AIDS among adolescents and the situation progress to date in addressing their needs for HIV prevention, care, and treatment services.

\section{Background and Significance}

Globally, an estimated 35.3 million people were living with HIV as at 2012, 2.1 million, of these estimated number were adolescents aged 10-19 years, of which the majority were girls accounting for $56 \%$ of the total 2.1 million estimated [5]. The gender disparity has persisted over time, with this number plateauing over the past years. According to literature, majority of this adolescent HIV burden, entirely $85.0 \%$ are resident in Sub-Saharan Africa. Trend analysis regarding HIV survival rates indicate that age-related HIV/AIDS mortality is reducing for all age groups except that of adolescents [7a]. [5], which reported that about 300,000 new infections occurred among adolescents aged 15-19 years, which accounted for about 13\% of the 2.3 million new infections globally. Along with the HIV/AIDS epidemic, a number of interventions and global goals have been agreed upon, notably to reduce the HIV prevalence in young people; to provide adequate information, life skills, health, and social services; and to implement policies to reduce young people's vulnerability [8]. Despite efforts, policy formulation and implementations, levels of knowledge of HIV among adolescents and young adults are appallingly low, especially in the worst affected countries; inconsistent compliance and poor practices of policies. Recent survey in countries with generalized epidemics [5], show that, in most of the countries, less than half of adolescent, aged 15-19 years, have a basic understanding of HIV. This falls far short of the 95\% target agreed in 2001 at the UNGASS. Reasonably, the vision 'ZERO New Infections; ZERO Deaths; ZERO Discrimination' formed by UNICEF for their 2020 target face the same fate. These differences persist in nearly all countries with available data [5]. Global and regional averages can mask individual country progress. A number of countries show data of enhanced knowledge about HIV prevention. For example, Belarus, Guyana, Jamaica, Namibia, Rwanda, Serbia, Swaziland, Trinidad and Tobago, Vietnam, and Zimbabwe witnessed significant increases in knowledge about HIV prevention to levels above 50\% among adolescent girls, and similar increases among adolescent boys in Rwanda and Namibia in the periods of 2010 and 2012 [7].

\section{The life of ALHIV}

ALHIV grow up in circumstances quite different from those of their other no-infected counterparts with both psychological, economic, social and sexuality challenge vulnerabilities 
[4]. They have an urgent need for improved approaches to address their specific health care needs [1]. Whiles several studies have explored in details the needs and challenges of adolescents [3],[4],[2], few studies have reported on the challenges ALHIV face in reality [9], [5]. Limited or no studies have explored the overall quality of life of ALHIV in terms of the fivefold needs in life (according to Abraham Maslow's theory of needs). Entirely all studies reporting on the life of ALHIV either talks about their social disadvantages, psychological vulnerabilities, environmental unfriendliness or compromised health status. Improving outcomes for adolescents and reaching global targets for an AIDS FREE generation by 2030 [10] will require evidence-based interventions and policies. Howbeit, there is a growing area of research in HIV and a considerable amount of efforts is still needed to inform the understanding of what works for this population.

Evidence indicates higher rates of loss to follow up [11], [12], and poor adherence [13], as well as increased needs for psychosocial support among ALHIV [14]. This population group continues to be underserved by current HIV services and have significantly worse access to and coverage of ART [6]. A number of care models have been designed over the years to improve health outcomes among this group of population.

\section{HIV testing and Disclosure models}

HIV testing is the key entry point for many HIV prevention interventions and is crucial for access to care and treatment [15]. Irrespective of route of HIV acquisition, underutilization of testing and counselling services (HTC) results in late diagnosis; increasing uptake of HTC consequently leads to early diagnosis and more effective care. Although reporting data on adolescents receiving treatment is limited, adolescents' access to and uptake of treatment is often reported to be lower compared to other age groups [16], [13]. It is urgent that ALHIV are recognized and signed-up in treatment interventions with clear and consistent linkages to care and support. Due to the increasing availability of antiretroviral therapy (ART) and prevention interventions, early diagnosis and linkage to care is able to reduce transmission and improve health outcomes [13], thereby decreasing adolescent morbidity and mortality. Although adolescents represent one of the wildest rising segments of the population to acquire HIV with diverse needs from those of children and adults, there is paucity of data focusing on this group [15]. Despite the fact that there are guidance on provider initiated testing and counselling (PITC), Voluntary Counselling Test (VCT) and prevention of mother-to-child transmission (PMTCT), these guidelines do not focus specifically on adolescents' issues [5], [17]. The development of WHO guidelines on adolescent HIV testing and counselling serves to fill an important gap which plays a critical role in prevention and treatment efforts [7a]. Howbeit guidelines recommended to assist policy makers to adapt to strategies for HTC among adolescents, cognitive, personal and a regulatory framework on informed consent based on adolescents' right; health-care decision-making requiring individuals to exercise their right to independent decision-making, may limit HTC service diversity and generalization.

Generally, studies of adolescents have found that disclosure has a significant association with improved clinical outcomes as measured by increased CD4 cell counts [18], decreased number of partners [19], and better linkage to care and adherence [20]. Disclosure decisions are often made to tell everyone (making HIV status a central attribute of one's identity), no one (requiring strategies for securing social support while remaining anonymous) or some people (requiring strategic decisions based on context) [21]. Knowing that disclosure decisions are central to personal identity, disclosure policies and strategies and as well as future researches should focus on communication strategies to present a coherent identity. Several studies have suggested disclosure strategies at different sites across different level including group counselling [22], structured support groups [23], one-on-one counselling, peer-led counselling 
[24], community-based interventions with disclosure component [25], individual counselling based intervention [26], and online computer or mobile phone base intervention [27].

\section{ART initiation and Linkage to care models}

Following HIV diagnosis, several studies [13], [28] revealed that linkage to outpatient treatment, antiretroviral initiation, and longitudinal retention in care characterize the basis for successful management. Effective establishment of HIV treatment demands that HIV diagnosis is followed by timely linkage to outpatient care, prompt initiation of ART and prophylactic medications when indicated, and subsequent adherence to prescribed medications as revealed in individual studies [29], [30], [31]. Certainty of uptake and successful outcomes demands that, patients must progress through a sequence of steps taking into account environmental, patients predisposing factors, perceived and enabling factors as well as health behaviour models as proposed in a study by Ulett et al. [28].

Unfortunately for ALHIV, studies reporting on ART initiation following diagnosis and linkage to care have its bases and concept not specific to adolescents. On the demands of ART initiation programs and subsequent long-term retention and successful outcome as reported by Ulett et al.[28], the contributing environmental, personal and health models is not well documented among ALHIV. However, the emphasis rely more on adults and children [32], [12]. A study conducted in the USA by [33] highlighted areas that could be explored for targeted interventions resulting in earlier diagnosis, ART initiation, improved adherence and retention in care among HIV-infected adolescents and young adults. They conclude that although there have been significant developments in HIV prevention with pre-exposure prophylaxis (PrEP) and treatment as prevention for adults, these interventions have not been sufficiently investigated, tailored, or scaled for youth. Their cascade highlighted that current efforts for treating already-infected adolescents and young adults remain a challenge. Most interventions to address the cascade have been developed for adults. These are not particularly generalizable to youth struggling with identity formation, economic hardships, and unstable housing. Youthfocused interventions are necessary to improve the HIV cascade for adolescents and young adults.

\section{Management and treatment models for ALHIV}

A combination of factors interdependent on themselves comes to play when conceptualizing and contextualizing management models for ALHIV (Figure 1). 


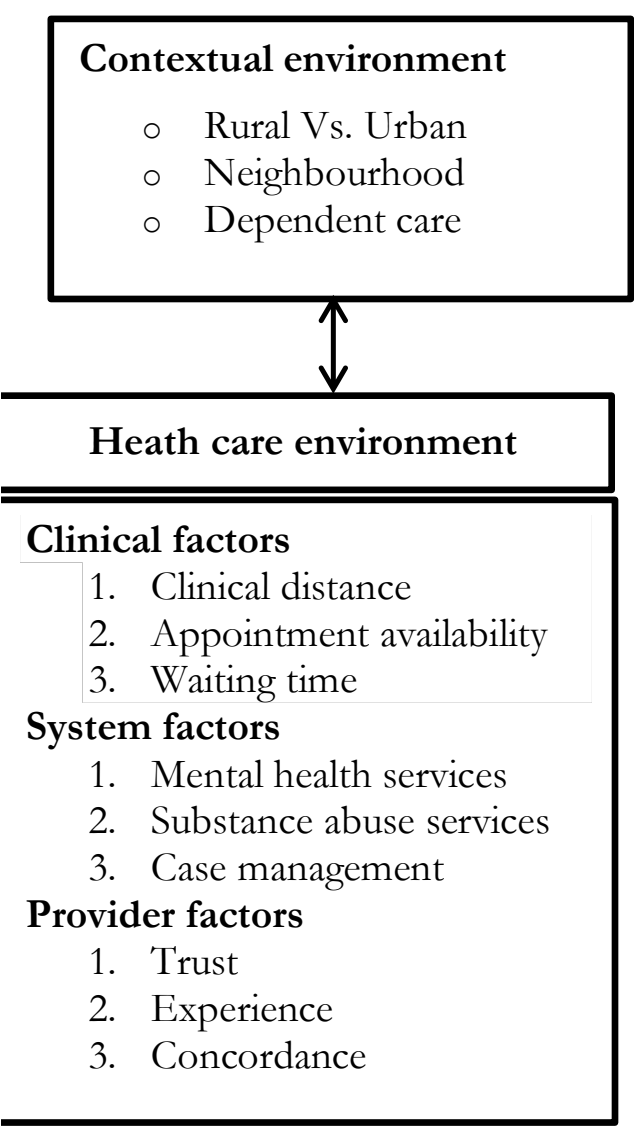

\section{Environment}

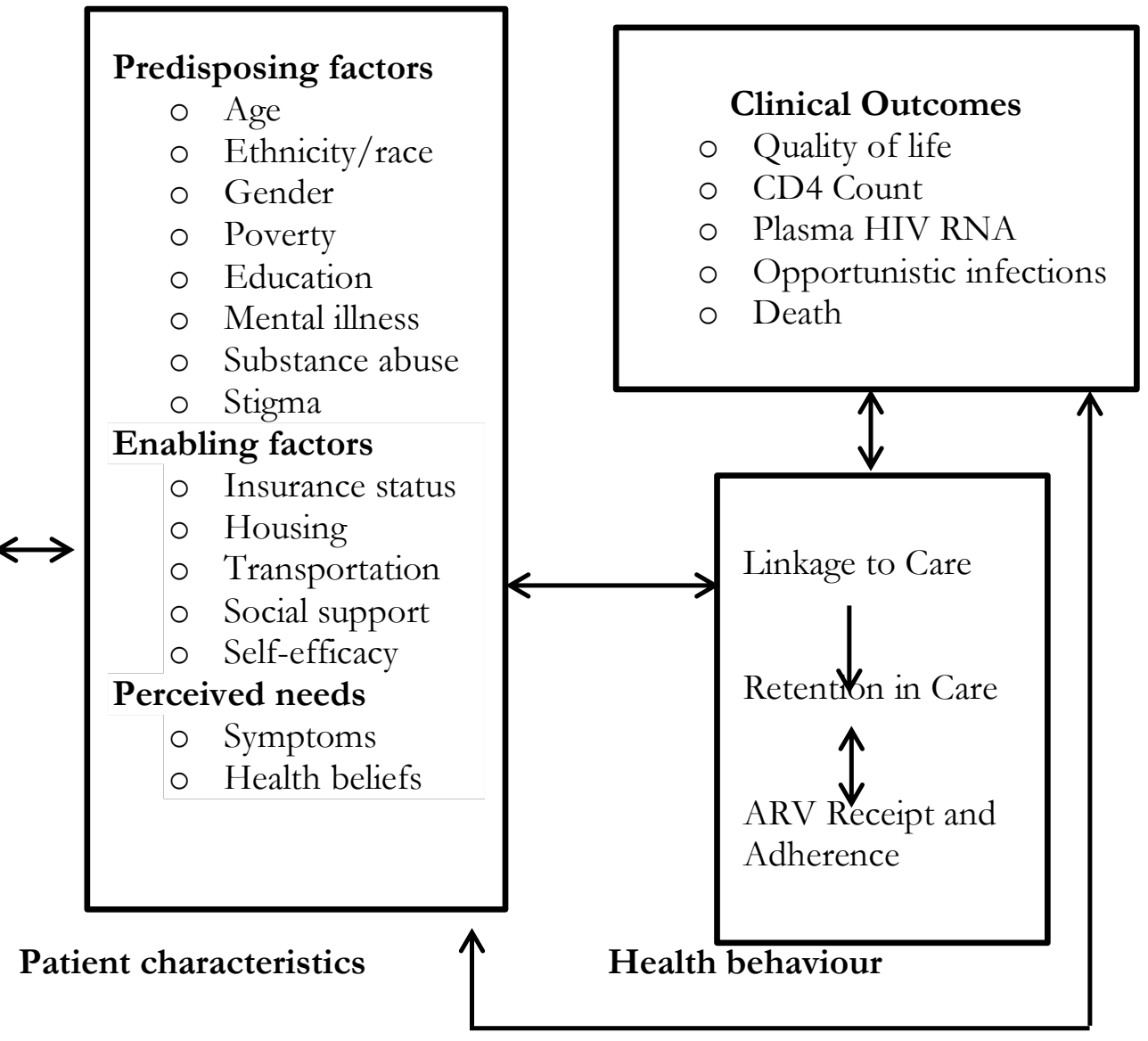

Fig 1.2 Adaptation of the behavioural model of health services operation to provide a theoretical framework to evaluate the relationships between patient characteristics, their background and health care environmental factors in contributing to health behaviours outlined in the "HIV management success" that ultimately influence clinical outcomes.

\section{Source: Adapted from Ulett et al. (2009)}

From literature search and WHO recommendations, two major intervention programs to support adherence to ART and retention in care included training of health workers and a range of community-based approaches. Adolescent management and care models for ALHIV happen to be one of the major areas mostly explored by researchers. Suggestions for improvements in services for ALHIV included age-appropriate support, material support (clothing, food, support for orphans), more protection from the damaging effects of stigma and discrimination, more comprehensive information about all the ways that HIV is transmitted, dedicated spaces and activities for ALHIV where they can be with peers who understand what it is like to live with HIV, and with educational opportunities for those who do not attend school [7a], [7b]. However despite effort made in the area of ALHIV care and management, agedisaggregated data on coverage of antiretroviral treatment (ART) among adolescents are lacking. Retaining adolescents in care is particularly challenging. Dealing with the prospect of lifelong treatment is daunting at any time in life, but for adolescent this comes on top of piloting the usual challenges of their developing maturity; emotionally, psychologically, physically, and sexually [5]. 


\section{Revised theoretical model from reviewing literature}

Despite significant HIV burden among adolescent and increased attention to integration of management services, there is uncertain evidence on the implementation of adolescent HIV management programs for adolescents living with HIV, nonetheless integrating such programs will improve outcome among ALHIV. Several studies have accounted for the successes of HIV clinics following the integration of secondary interventions such as peer support groups, mobile phone sections, community interventions [8], Adolescent Coordinated Transition (ACT) models [34] and family centred models [35].

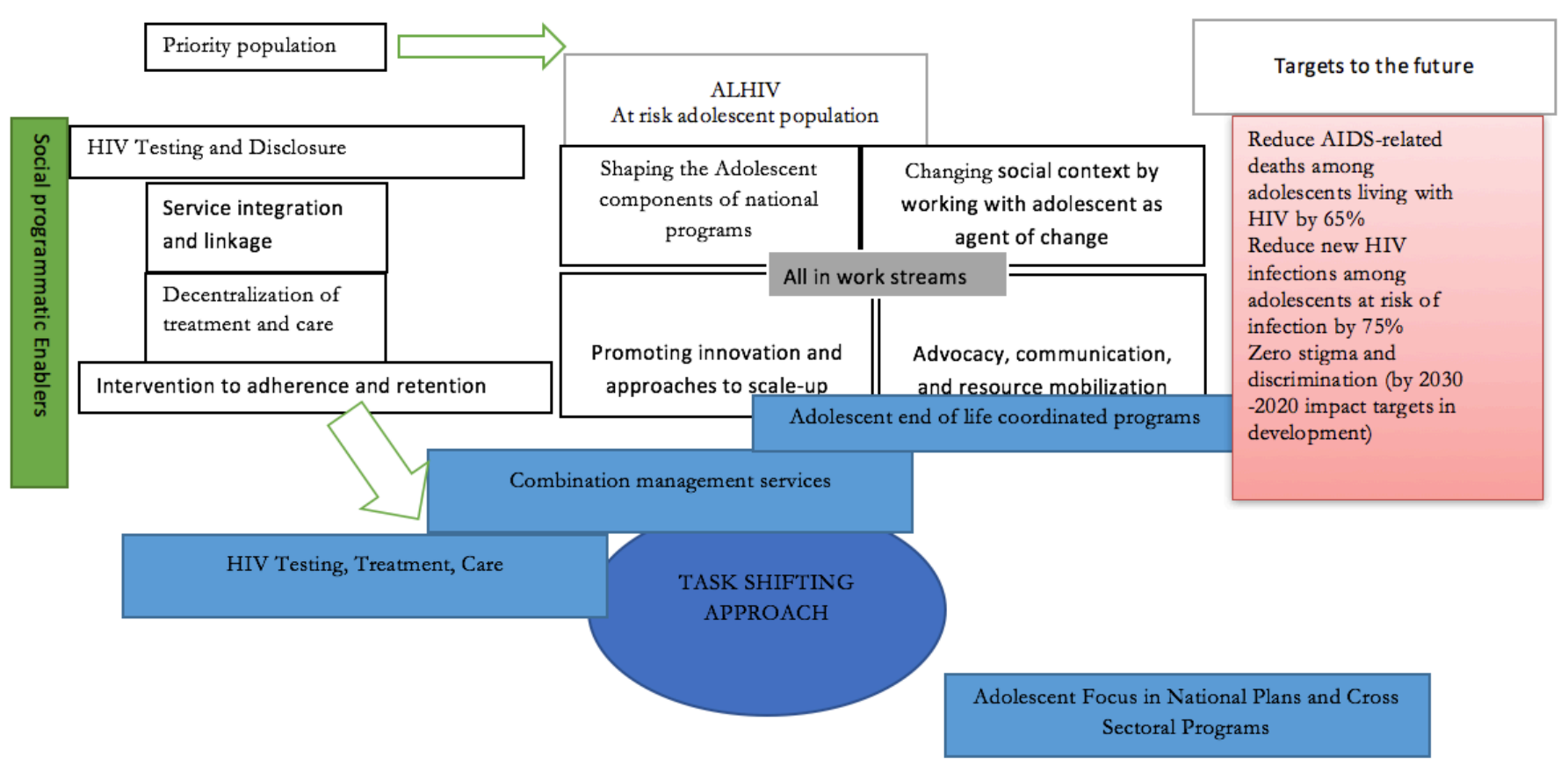

Figure 1.1: All in one strategic management model for ALHIV

\section{RECOMMENDATIONS AND FUTURE DIRECTIONS}

The physiognomies and requirements of adolescents cannot be addressed by applying guidelines based on evidence and recommendations relating to paediatric or adult populations. Moreover, adolescents themselves are not a homogeneous group. Physical and psychological (cognitive and emotional) development varies among adolescents, and differing social and cultural factors as well as their evolving capacities can affect both their ability to make important personal decisions and their access to services. For these reasons guidelines should take into consideration the range of adolescents' needs and issues surrounding their health. Again Stakeholders involved in clinical and operational research and policy formulation in adolescent HIV care can support the successful implementation of this task by actively engaging the adolescent and the established priorities and integrating them into their activities for adolescents.

\section{References}

Hindin M.J. and Fatusi A.O. (2009) Adolescent sexual and reproductive health in developing countries: an overview of trends and interventions. International perspectives on sexual and reproductive health 35(2), 58-62.

Leversen I., Danielsen A.G., Birkeland M.S. and Samdal O. (2012) Basic psychological need satisfaction in leisure activities and adolescents' life satisfaction. Journal of Youth and Adolescence 41(12), 1588-1599.

Habermas T. and Paha C. (2001) The development of coherence in adolescents' life narratives. Narrative Inquiry 11(1), 35-54.

Bearinger L.H., Sieving R.E., Ferguson J. and Sharma V. (2007) Global perspectives on the sexual and reproductive health of adolescents: patterns, prevention, and potential. The lancet 369(9568), 1220-1231. 
Idele P., Gillespie A., Porth T., Suzuki C., Mahy M., Kasedde S. and Luo C. (2014) Epidemiology of HIV and AIDS among adolescents: current status, inequities, and data gaps. JAIDS Journal of Acquired Immune Deficiency Syndromes 66S144-S153.

Organization W.H. (2017) Research for and AIDS free generation: a global research agenda for adolescents living with HIV.

Organization W.H. (2013a) Global update on HIV treatment 2013: results, impact and opportunities.

Organization W.H. (2013b) HIV and adolescents: Guidance for HIV testing and counselling and care for adolescents living with HIV: Recommendations for a public health approach and considerations for policy-makers and managers.

Nabukeera-Barungi N., Elyanu P., Asire B., Katureebe C., Lukabwe I., Namusoke E., Musinguzi J., Atuyambe L. and Tumwesigye N. (2015) Adherence to antiretroviral therapy and retention in care for adolescents living with HIV from 10 districts in Uganda. BMC infectious diseases 15(1), 520.

Sampaio F.J.d.L.S., do Amaral Gubert F., da Costa Pinheiro P.N., Martins A.K.L., Vieira N.F.C. and Nóbrega M.d.F.B. (2013) The life of the adolescent with HIV/AIDS and self-care: A descriptive study. Online Brazilian Journal of Nursing 12(1), 89-105.

Organization W.H. (2015) Report of the consultation on the treatment of HIV among adolescents. Geneva: WHO.

Auld A.F., Agolory S.G., Shiraishi R.W., Wabwire-Mangen F., Kwesigabo G., Mulenga M., Hachizovu S., Asadu E., Tuho M.Z. and Ettiegne-Traore V. (2014) Antiretroviral therapy enrollment characteristics and outcomes among HIV-infected adolescents and young adults compared with older adults-seven African countries, 2004-2013. Morbidity and Mortality Weekly Report 63(47), 1097-1103.

Lamb M.R., Fayorsey R., Nuwagaba-Biribonwoha H., Viola V., Mutabazi V., Alwar T., Casalini C. and Elul B. (2014) High attrition before and after ART initiation among youth (15-24 years of age) enrolled in HIV care. AIDS (London, England) 28(4), 559.

Nachega J.B., Hislop M., Nguyen H., Dowdy D.W., Chaisson R.E., Regensberg L., Cotton M. and Maartens G. (2009) Antiretroviral therapy adherence, virologic and immunologic outcomes in adolescents compared with adults in southern Africa. Journal of acquired immune deficiency syndromes (1999) 51(1), 65.

Denison J.A., Banda H., Dennis A.C., Packer C., Nyambe N., Stalter R.M., Mwansa J.K., Katayamoyo P. and McCarraher D.R. (2015) "The sky is the limit": adhering to antiretroviral therapy and HIV self-management from the perspectives of adolescents living with HIV and their adult caregivers. Journal of the International AIDS Society 18(1).

HIV W. (2013) adolescents: guidance for HIV testing and counselling and care for adolescents living with HIV: recommendations for a public health approach and considerations for policy-makers and managers. Geneva, Switzerland: World Health Organization.

Lindsey J.C., Bosch R.J., Rudy B.J. and Flynn P.M. (2009) Early patterns of adherence in adolescents initiating highly active antiretrovial therapy predict long-term adherence, virologic, and immunologic control. AIDS patient care and STDs 23(10), 799-801.

Schouten E., Sinunu M., Wadonda N., Kajawo E., Eliya M., Chimbwandira F. and Kellerman S. (2012) More HIV positive infants and mothers identified through HIV testing in immunization clinics. Journal of the International AIDS Society 15, 110-110.

Sherman B.F., Bonanno G.A., Wiener L.S. and Battles H.B. (2000) When children tell their friends they have AIDS: possible consequences for psychological well-being and disease progression. Psychosomatic medicine 62(2), 238247.

Dempsey A.G., MacDonell K.E., Naar-King S. and Lau C.-Y. (2012) Patterns of disclosure among youth who are HIVpositive: a multisite study. Journal of Adolescent Health 50(3), 315-317.

Beyene K.A., Gedif T., Gebre-Mariam T. and Engidawork E. (2009) Highly active antiretroviral therapy adherence and its determinants in selected hospitals from south and central Ethiopia. Pharmacoepidemiology and drug safety 18(11), 1007-1015.

Mayfield Arnold E., Rice E., Flannery D. and Rotheram-Borus M.J. (2008) HIV disclosure among adults living with HIV. AIDS care 20(1), 80-92.

Fawzi M.C.S., Eustache E., Oswald C., Louis E., Surkan P.J., Scanlan F., Hook S., Mancuso A. and Mukherjee J.S. (2012) Psychosocial support intervention for HIV-affected families in Haiti: implications for programs and policies for orphans and vulnerable children. Social science \& medicine 74(10), 1494-1503. 
Murphy D.A., Armistead L., Marelich W.D., Payne D.L. and Herbeck D.M. (2011) Pilot trial of a disclosure intervention for HIV+ mothers: The TRACK program. Journal of Consulting and Clinical Psychology 79(2), 203.

Wolitski R.J., Gómez C.A., Parsons J.T. and Group S.S. (2005) Effects of a peer-led behavioral intervention to reduce HIV transmission and promote serostatus disclosure among HIV-seropositive gay and bisexual men. Aids 19S99S109.

Dewo Z., Tollera G., Trivelli M. and Gebremariam M. (2012) Strengthening treatment, care and support to people living with HIV through community-based treatment services. Journal of the International AIDS Society 15, 205205.

Patterson T.L., Shaw W.S. and Semple S.J. (2003) Reducing the sexual risk behaviors of HIV+ individuals: outcome of a randomized controlled trial. Annals of Behavioral Medicine 25(2), 137-145.

Chiasson M.A., Shaw F.S., Humberstone M., Hirshfield S. and Hartel D. (2009) Increased HIV disclosure three months after an online video intervention for men who have sex with men (MSM). AIDS care 21(9), 1081-1089.

Ulett K.B., Willig J.H., Lin H.-Y., Routman J.S., Abroms S., Allison J., Chatham A., Raper J.L., Saag M.S. and Mugavero M.J. (2009) The therapeutic implications of timely linkage and early retention in HIV care. AIDS patient care and STDs 23(1), 41-49.

Janssen R.S., Holtgrave D.R., Valdiserri R.O., Shepherd M., Gayle H.D. and De Cock K.M. (2001) The serostatus approach to fighting the HIV epidemic: prevention strategies for infected individuals. American journal of public health 91(7), 1019.

Cheever L.W. (2007) Engaging HIV-infected patients in care: their lives depend on it: The University of Chicago Press.

Giordano T.P., Gifford A.L., White Jr A.C., Almazor M.E.S., Rabeneck L., Hartman C., Backus L.I., Mole L.A. and Morgan R.O. (2007) Retention in care: a challenge to survival with HIV infection. Clinical infectious diseases 44(11), 1493-1499.

Bradley H., Hall H.I., Wolitski R.J., Van Handel M.M., Stone A.E., LaFlam M., Skarbinski J., Higa D.H., Prejean J. and Frazier E.L. (2014) Vital signs: HIV diagnosis, care, and treatment among persons living with HIV-United States, 2011. Morbidity and Mortality Weekly Report 63(47), 1113-1117.

Zanoni B.C. and Mayer K.H. (2014) The adolescent and young adult HIV cascade of care in the United States: exaggerated health disparities. AIDS patient care and STDs 28(3), 128-135.

Sam-Agudu N.A., Pharr J.R., Bruno T., Cross C.L., Cornelius L.J., Okonkwo P., Oyeledun B., Khamofu H., Olutola A. and Erekaha S. (2017) Adolescent Coordinated Transition (ACT) to improve health outcomes among young people living with HIV in Nigeria: study protocol for a randomized controlled trial. Trials 18(1), 595.

Wiener L. and Rosenberg A.R. (2017) Family- centred advanced care planning with adolescents living with HIV is perceived as important, helpful and meaningful. Evidence-based nursingebnurs-2017-102683. 